\title{
Flexible Multibody Systems Models Using Composite Materials Components
}

\author{
MARIA AUGUSTA NETO ${ }^{1}$, JORGE A.C. AMBRÓSIO ${ }^{2}$ and ROGÉRIO \\ PEREIRA LEAL ${ }^{3}$ \\ ${ }^{1,3}$ Departamento de Engenharia Mecânica - Faculdade de Ciência e Tecnologia da Universidade de \\ Coimbra (Polo II), 3020 Coimbra, Portugal \\ ${ }^{2}$ Instituto de Engenharia Mecânica, Instituto Superior Técnico, Av. Rovisco Pais, 1049-001 Lisboa, \\ Portugal; \\ E-mail:jorge@dem.ist.utl.pt
}

(Received: 01 September 2003; accepted in revised form 13 May 2004)

\begin{abstract}
The use of a multibody methodology to describe the large motion of complex systems that experience structural deformations enables to represent the complete system motion, the relative kinematics between the components involved, the deformation of the structural members and the inertia coupling between the large rigid body motion and the system elastodynamics. In this work, the flexible multibody dynamics formulations of complex models are extended to include elastic components made of composite materials, which may be laminated and anisotropic. The deformation of any structural member must be elastic and linear, when described in a coordinate frame fixed to one or more material points of its domain, regardless of the complexity of its geometry. To achieve the proposed flexible multibody formulation, a finite element model for each flexible body is used. For the beam composite material elements, the sections properties are found using an asymptotic procedure that involves a two-dimensional finite element analysis of their cross-section. The equations of motion of the flexible multibody system are solved using an augmented Lagrangian formulation and the accelerations and velocities are integrated in time using a multi-step multi-order integration algorithm based on the Gear method.
\end{abstract}

Key words: composite material, flexible multibody systems, elastic coupling, mode component synthesis

\section{Introduction}

The need for more accurate models to describe the complex behavior of flexible systems experiencing large motion while undergoing small elastic deformations motivated the development of many powerful analysis techniques. The most popular formulations use time-variant mass matrices to describe the inertia coupling between the rigid body gross motion and the system elastodynamics [1]. Some coefficients of these inertia coupling matrices are dependent on the type of finite elements used in the model. They do not appear in standard finite element developments and consequently need to be specially derived when these methodologies 
are used. A procedure proposed by Ambrósio and Gonçalves [2] allows for these coupling matrices to be evaluated in a pre-processing stage and to eliminate its dependency in the type of finite element used in the model.

The finite element formulation for the flexible bodies use a body fixed coordinate frame to describe the deformation field of each body. The flexible bodies together with the rigid bodies of the multibody system are represented by a set of Cartesian coordinates and have their relative motion restrained by a set of kinematic constraints [3, 4]. Other formulations for the multibody system such as the natural coordinates [5] can also be used with the finite element description of the large motion of the flexible bodies. However, for any of the possible set of coordinates that can be used it is necessary to define the kinematic constraints between the flexible and the rigid bodies of the system. The description based on the use of virtual bodies is applied in this work [6].

The formulations used for the description of large motion of flexible members have been used in the framework of systems made of standard materials. Recently, efforts have been made to describe composite and laminated materials in the framework of multibody systems [7]. The work now presented is a contribution to the representation of flexible multibody systems using composite materials. In particular, one method to describe composite beam elements that follows the work proposed by Cesnik and Hodges [8] is presented and another technique to represent plate/shell elements with orthotropic lamina. This methodology is applied to demonstrative examples, where the flexibility of members of a multibody system plays a decisive role in the behavior of the system.

\section{Multibody Formulation and Equations of Motion}

\subsection{FLEXIBLE BODY MOTION}

Let it be assumed that a flexible body is composed of a coordinate system rigidly attached to a point on the flexible component, as depicted by Figure 1. Let it also be assumed that the flexible body is represented using the finite element method, with a lumped formulation for the mass matrix, obtained by the diagonalization of the consistent mass matrix. The reference frame, located by vector $\mathbf{r}_{i}$ with respect to the inertia frame is fixed to the body center of mass [1].

The flexible body kinetic energy is expressed as

$$
T_{i}=\frac{1}{2} \dot{\mathbf{q}}_{i}^{\mathrm{T}} \mathbf{M}_{i} \dot{\mathbf{q}}_{i}
$$

where the velocity vector for a single flexible body $i$, is represented by $\dot{\mathbf{q}}_{i}=$ $\left[\dot{\mathbf{r}}_{i}^{\mathrm{T}} \boldsymbol{\omega}_{i}^{\mathrm{T}} \dot{\mathbf{u}}_{i}^{\mathrm{T}}\right]^{\mathrm{T}}$. The velocity vector contains the rigid body velocities $\dot{\mathbf{q}}_{r_{i}}=\left[\dot{\mathbf{r}}_{i}^{\mathrm{T}} \boldsymbol{\omega}_{i}^{\mathrm{T}}\right]^{\mathrm{T}}$ and the vector of the local nodal velocities $\dot{\mathbf{u}}_{i}^{\prime}=\left[\begin{array}{ll}\dot{\boldsymbol{\delta}}^{\prime \mathrm{T}} & \dot{\boldsymbol{\theta}}^{\prime \mathrm{T}}\end{array}\right]_{i}^{\mathrm{T}}$. The flexible body 


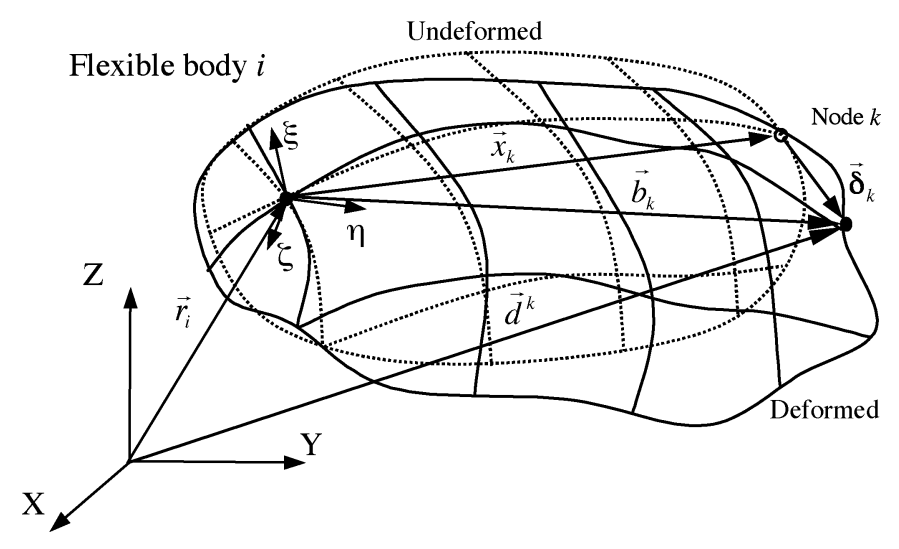

Figure 1. Global position of node $k$.

mass matrix $\mathrm{M}_{i}$ is given by

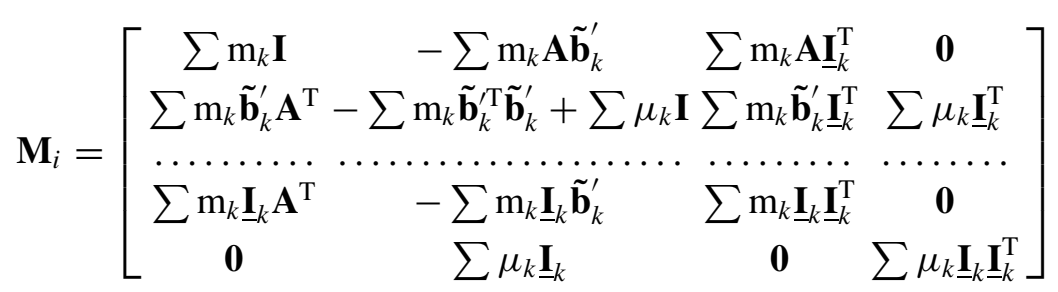

In methods where the consistent mass formulation is used in the finite element description of the flexible body, the inertia coupling terms depend on the particular finite element shape functions used. The structure of the mass matrix given by Equation (2) is independent of the formulation used to describe the flexible body deformations and no special inertia-coupling coefficients have to be derived.

The elastic energy for the flexible body, expressed by $U$, is written as

$$
\mathrm{U}=\frac{1}{2} \mathbf{q}^{\mathrm{T}} \underline{\mathbf{K}} \mathbf{q}
$$

where $\underline{\mathbf{K}}$ is an augmented stiffness matrix written as

$$
\underline{\mathbf{K}}=\left[\begin{array}{cc}
\mathbf{0} & \mathbf{0} \\
\mathbf{0} & \mathbf{K}_{f f}
\end{array}\right]
$$

and where $\mathbf{K}_{f f}$ is the standard finite element stiffness matrix or any other equivalent matrix, depending on the adopted description of the body flexibility. However, if matrix $\mathbf{K}_{f f}$ is not dependent on the deformation of the flexible body, the formulation implies that only linear elastic deformations are assumed. This is the case for all that follows. 
As all coordinates defined in Equation (1) are independent, the Lagrange equations of motion for the flexible body are given by

$$
\frac{\mathrm{d}}{\mathrm{d} t}\left(\frac{\partial T_{i}}{\partial \dot{\mathbf{q}}_{i}}\right)-\left(\frac{\partial T_{i}}{\partial \mathbf{q}_{i}}\right)+\left(\frac{\partial U_{i}}{\partial \mathbf{q}_{i}}\right)-\mathbf{g}_{i}=\mathbf{0}
$$

The definitions of the kinetic and elastic energy, given by Equations (2) and (3) respectively, are now substituted in Equation (5) leading to

$$
\mathbf{M}_{i} \ddot{\mathbf{q}}_{i}=\mathbf{g}_{i}+\mathbf{s}_{i}-\underline{\mathbf{K}_{i}} \mathbf{q}_{i}
$$

where $\mathbf{g}_{i}$ is the vector of external applied forces, $\mathbf{s}_{i}$ is given by

$$
\mathbf{s}_{i}=\left[\begin{array}{c}
-\sum m_{k} \mathbf{A}_{i} \tilde{\boldsymbol{\omega}}_{i}^{\prime} \tilde{\boldsymbol{\omega}}_{i}^{\prime} \mathbf{b}_{i}^{\prime k}-2 \sum m_{k} \mathbf{A}_{i} \tilde{\boldsymbol{\omega}}_{i}^{\prime} \dot{\boldsymbol{\delta}}_{i}^{\prime k} \\
-\sum m_{k} \tilde{\boldsymbol{\omega}}_{i}^{\prime} \tilde{\mathbf{b}}_{i}^{\prime k} \tilde{\boldsymbol{\omega}}_{i}^{\prime} \mathbf{b}_{i}^{\prime k}-2 \sum m_{k} \tilde{\mathbf{b}}_{i}^{\prime k} \tilde{\boldsymbol{\omega}}_{i}^{\prime} \dot{\boldsymbol{\delta}}_{i}^{k}-\sum m_{k} \tilde{\boldsymbol{\omega}}_{i}^{\prime} \tilde{\mathbf{b}}_{i}^{\prime k} \mathbf{I}_{i}^{\mathrm{T}} \dot{\boldsymbol{\delta}}_{i}^{\prime k} \\
-\sum m_{k} \mathbf{I}_{i}^{k} \tilde{\boldsymbol{\omega}}_{i}^{\prime} \tilde{\boldsymbol{\omega}}_{i}^{\prime} \mathbf{b}_{i}^{\prime k}-2 \sum m_{k} \mathbf{I}_{i}^{k} \tilde{\boldsymbol{\omega}}_{i}^{\prime} \dot{\boldsymbol{\delta}}_{i}^{k} \\
\mathbf{0}
\end{array}\right]
$$

and it contains the quadratic velocity terms.

\subsection{SYSTEM EQUATIONS OF MOTION}

For a rigid and flexible multibody system, it is necessary to define a set of kinematic constraints describing the joints that restrict the relative motion between the bodies of the system, what implies that not all coordinates are independent. These kinematic constraints are defined by a set of algebraic equations and can be added to the Lagrange equations by using a vector of Lagrange multipliers, $\lambda$. These are written as

$$
\frac{\mathrm{d}}{\mathrm{dt}}\left(\frac{\partial \mathrm{T}}{\partial \dot{\mathbf{q}}}\right)-\left(\frac{\partial \mathrm{T}}{\partial \mathbf{q}}\right)+\left(\frac{\partial \mathrm{U}}{\partial \mathbf{q}}\right)+\boldsymbol{\Phi}_{\mathbf{q}}^{\mathrm{T}} \boldsymbol{\lambda}-\mathbf{g}=\mathbf{0}
$$

which leads to

$$
\mathbf{M} \ddot{\mathbf{q}}+\Phi_{\mathbf{q}}^{\mathrm{T}} \lambda=\mathbf{g}+\mathbf{s}-\underline{\mathbf{K q}}
$$

The constraints acceleration equations are given by

$$
\ddot{\Phi}\left(\mathbf{q}_{r}, \mathbf{u}^{\prime}, t\right) \equiv \boldsymbol{\Phi}_{\mathbf{q}} \ddot{\mathbf{q}}-\gamma^{*}=\mathbf{0}
$$

the vector $\gamma^{*}$ is used to represent all terms independent of the acceleration, as

$$
\gamma^{*}=-\left(\Phi_{\mathbf{q}} \dot{\mathbf{q}}\right)_{\mathbf{q}} \dot{\mathbf{q}}-\Phi_{t t}-2 \Phi_{\mathbf{q}} \dot{\mathbf{q}}
$$


adding the Equation (10) to the system equations of motion, leads to

$$
\left[\begin{array}{ccc}
\mathbf{M}_{r} & \mathbf{M}_{r f} & \boldsymbol{\Phi}_{\mathbf{q}_{r}}^{\mathrm{T}} \\
\mathbf{M}_{f r} & \mathbf{M}_{f f} & \boldsymbol{\Phi}_{\mathbf{u}^{\prime}}^{\mathrm{T}} \\
\boldsymbol{\Phi}_{\mathbf{q}_{r}} & \boldsymbol{\Phi}_{\mathbf{u}^{\prime}} & \mathbf{0}
\end{array}\right]\left\{\begin{array}{c}
\ddot{\mathbf{q}}_{r} \\
\ddot{\mathbf{u}}^{\prime} \\
\boldsymbol{\lambda}
\end{array}\right\}=\left\{\begin{array}{c}
\mathbf{g}_{r} \\
\mathbf{g}_{f} \\
\gamma^{*}
\end{array}\right\}-\left\{\begin{array}{c}
\mathbf{s}_{r} \\
\mathbf{s}_{f} \\
\mathbf{0}
\end{array}\right\}-\left\{\begin{array}{c}
\mathbf{0} \\
\mathbf{K}_{f f} \mathbf{u}^{\prime} \\
\mathbf{0}
\end{array}\right\}
$$

Second-order equations, such as Equation (12), are unstable. Small perturbations, such as the numerical errors introduced by the integration process, cannot be corrected naturally and they only tend to be amplified. The solution is to introduce feedback terms that penalize the system response, if violations on the position or velocity constraint equations occur. With this purpose in mind, the right-hand side of Equation (11) is modified using the Baumgarte method [3]

$$
\gamma=\gamma^{*}-2 \alpha \dot{\boldsymbol{\Phi}}-\beta^{2} \boldsymbol{\Phi}
$$

where the $\alpha$ and $\beta$ are positive constants that weight the violations of the velocity and position constraint equations respectively. These constants, for a multibody system made of rigid and flexible bodies, are values in the range of $1-10$, being $\alpha, \beta=5$ values often used [3].

Replacing the vector $\gamma^{*}$ in Equation (12) by the vector $\gamma$ leads to

$$
\left[\begin{array}{ccc}
\mathbf{M}_{r} & \mathbf{M}_{r f} & \boldsymbol{\Phi}_{\mathbf{q}_{r}}^{\mathrm{T}} \\
\mathbf{M}_{f r} & \mathbf{M}_{f f} & \boldsymbol{\Phi}_{\mathbf{u}^{\prime}}^{\mathrm{T}} \\
\boldsymbol{\Phi}_{\mathbf{q}_{r}} & \boldsymbol{\Phi}_{\mathbf{u}^{\prime}} & \mathbf{0}
\end{array}\right]\left\{\begin{array}{c}
\ddot{\mathbf{q}}_{r} \\
\ddot{\mathbf{u}}^{\prime} \\
\boldsymbol{\lambda}
\end{array}\right\}=\left\{\begin{array}{c}
\mathbf{g}_{r} \\
\mathbf{g}_{f} \\
\gamma
\end{array}\right\}-\left\{\begin{array}{c}
\mathbf{s}_{r} \\
\mathbf{s}_{f} \\
\mathbf{0}
\end{array}\right\}-\left\{\begin{array}{c}
\mathbf{0} \\
\mathbf{K}_{f f} \mathbf{u}^{\prime} \\
\mathbf{0}
\end{array}\right\}
$$

The derivation of kinematic joints involving one or more flexible bodies is generally a complex task that must be repeated for different sets of flexible coordinates. The virtual bodies are applied in the definition of the joints that involve flexible bodies, requiring only for a rigid joint to be derived between flexible and rigid/virtual bodies [6]. This is the approach followed in the models presented in this work.

\subsection{COORDINATES REDUCTION BY COMPONENT MODE SYNTHESIS}

The equations of motion for the flexible multibody systems, in the form described by Equation (10) lead to an inefficient numerical implementation, due to the large number of generalized coordinates necessary to describe complex models. This problem is overcome by using a component mode synthesis methodology [2]. Although, only the modes of vibration are used in this formulation, other modes such as the static correction modes $[9,10]$ can be considered in order to improve numerical precision and efficiency. In the same manner, the use of the mean axis condition [11], can also be considered. However, in what follows only body-fixed coordinate systems are considered, and therefore, only constrained modes are used. 
Let the nodal displacements of the flexible part of the body be described by a weighted sum of the modes of vibration associated with the flexible bodies natural frequencies

$$
\mathbf{u}^{\prime}=\mathbf{X} \mathbf{w}
$$

where the vector $\mathbf{w}$ represents the contributions of the vibration modes towards the nodal displacements and $\mathbf{X}$ is the modal matrix. Due to the reference conditions, the modes of vibration used in this formulation are constrained modes. Moreover, due to the assumption of linear elastic deformations the modal matrix is invariant. The application of the orthonormality of the modes of vibration with respect to the mass matrix, i.e. $\mathbf{X}^{\mathrm{T}} \mathbf{M}_{f f} \mathbf{X}=\mathbf{I}$ and $\mathbf{X}^{\mathrm{T}} \mathbf{K}_{f f} \mathbf{X}=\boldsymbol{\Lambda}$, where $\boldsymbol{\Lambda}$ is a diagonal matrix containing the squares of the flexible body natural frequencies, leads to a simpler system of equations written as

$$
\left[\begin{array}{ccc}
\mathbf{M}_{r} & \mathbf{M}_{r f} \mathbf{X} & \boldsymbol{\Phi}_{\mathbf{q}_{r}}^{\mathrm{T}} \\
\mathbf{X}^{T} \mathbf{M}_{f r} & \mathbf{I} & \mathbf{X}^{\mathrm{T}} \boldsymbol{\Phi}_{\mathbf{u}^{\prime}}^{\mathrm{T}} \\
\boldsymbol{\Phi}_{\mathbf{q}_{r}} & \boldsymbol{\Phi}_{\mathbf{u}^{\prime}}^{\mathbf{X}} & \mathbf{0}
\end{array}\right]\left\{\begin{array}{c}
\ddot{\mathbf{q}}_{r} \\
\ddot{\mathbf{u}}^{\prime} \\
\boldsymbol{\lambda}
\end{array}\right\}=\left\{\begin{array}{c}
\mathbf{g}_{r} \\
\mathbf{X}^{\mathrm{T}} \mathbf{g}_{f} \\
\boldsymbol{\gamma}
\end{array}\right\}-\left\{\begin{array}{c}
\mathbf{s}_{r} \\
\mathbf{X}^{\mathrm{T}} \mathbf{s}_{f} \\
\mathbf{0}
\end{array}\right\}-\left\{\begin{array}{c}
\mathbf{0} \\
\boldsymbol{\Lambda} \mathbf{u}^{\prime} \\
\mathbf{0}
\end{array}\right\}
$$

This is a much smaller set of equations for describing the flexible body than those implied by Equation (14). Instead of having close to six-times the number of the nodes of the finite element mesh as generalized coordinates, the actual system only involves as many generalized coordinates as modes of vibration. Depending on the structure that is modeled a few number of modes may be used, in comparison with the large number of nodal coordinates, to represent with accuracy the structural response. All terms required for Equation (16), related with a finite element model, are obtained directly from any commercial finite element code. These coefficients are the finite element mass matrix $\mathbf{M}_{f f}$, the modes of vibration used in the modal matrix $\mathbf{X}$ and the corresponding natural frequencies. Therefore, the finite element code supporting this methodology can be viewed as a pre-processor. Alternatively, experimental modes of vibration can be used with this procedure.

\section{Finite Elements with Flexible Bodies in Composite Materials}

Due to their geometries, rotor blades, wings, space frames, and many other structural components have one or two dimensions that are much larger than the other dimension(s). Such flexible structures can often be treated as beams or plate/shells. Laminated beams or plate/shells are presently used as structural elements in general high performance mechanical aerospace, naval and civil applications, where high strength and high stiffness to weight ratios are required [12]. In the approach proposed here, the flexibility is included in the multibody system assuming the hypothesis of small displacements and rotations with respect to a body attached 


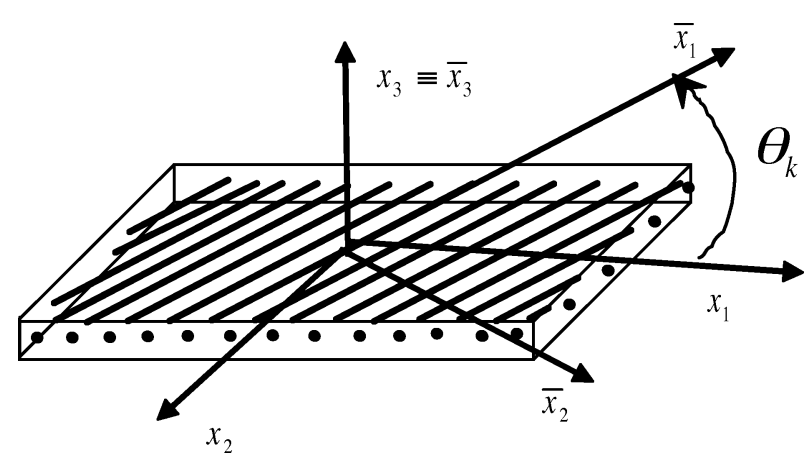

Figure 2. An orthotropic layer with principal material directions.

reference frame. Under such conditions, linear finite elements can also handle problems in a nonlinear elastic range.

\subsection{ORTHOTROPIC LAMINA}

Composite materials consisting of stiff and strong fibers embedded in a compatible matrix are named fibrous composites. Laminated composites are made with layers of different materials and/or different orientations. For a layer of orthotropic material represented in Figure 2, i.e., for which the material has three orthogonal planes of elastic symmetry, the constitutive equations in the material coordinate system, are written as [13]

$$
\left[\begin{array}{l}
\bar{\sigma}_{11} \\
\bar{\sigma}_{22} \\
\bar{\sigma}_{33} \\
\bar{\sigma}_{23} \\
\bar{\sigma}_{13} \\
\bar{\sigma}_{12}
\end{array}\right]_{k}=\left(\left[\begin{array}{cccccc}
\bar{c}_{11} & \bar{c}_{12} & \bar{c}_{13} & 0 & 0 & 0 \\
\bar{c}_{21} & \bar{c}_{22} & \bar{c}_{23} & 0 & 0 & 0 \\
\bar{c}_{31} & \bar{c}_{32} & \bar{c}_{33} & 0 & 0 & 0 \\
0 & 0 & 0 & \bar{c}_{44} & 0 & 0 \\
0 & 0 & 0 & 0 & \bar{c}_{55} & 0 \\
0 & 0 & 0 & 0 & 0 & \bar{c}_{66}
\end{array}\right]\left[\begin{array}{c}
\bar{\varepsilon}_{11} \\
\bar{\varepsilon}_{22} \\
\bar{\varepsilon}_{33} \\
\bar{\varepsilon}_{23} \\
\bar{\varepsilon}_{13} \\
\bar{\varepsilon}_{12}
\end{array}\right]\right)_{k}
$$

where indexes 1, 2 and 3 refer to the axis of the system that form the three orthogonal planes of material symmetry. The elastic coefficients $\bar{c}_{i j}$ in the Equation (17) are related with the engineering constants $E_{i}, v_{i j}$ and $G_{i j}$.

Generally the coordinate system used in the solution of the problem does not coincide with the material coordinate system. Furthermore, the composite laminates have several layers, each with a different orientation $\theta_{k}$ of their material coordinates with respect to the laminate coordinates. Thus, it is necessary to establish transformation relations among stresses and strains in one coordinate system to the corresponding quantities in another coordinate system. Upon transformation, the 
lamina constitutive equations are expressed in the global coordinate system as

$$
\left[\begin{array}{l}
\sigma_{11} \\
\sigma_{22} \\
\sigma_{33} \\
\sigma_{23} \\
\sigma_{13} \\
\sigma_{12}
\end{array}\right]_{k}=\left(\left[\begin{array}{cccccc}
c_{11} & c_{12} & c_{13} & 0 & 0 & c_{16} \\
c_{21} & c_{22} & c_{23} & 0 & 0 & c_{26} \\
c_{31} & c_{32} & c_{33} & 0 & 0 & c_{36} \\
0 & 0 & 0 & c_{44} & c_{45} & 0 \\
0 & 0 & 0 & c_{54} & c_{55} & 0 \\
c_{61} & c_{62} & c_{63} & 0 & 0 & c_{66}
\end{array}\right]\left[\begin{array}{c}
\varepsilon_{11} \\
\varepsilon_{22} \\
\varepsilon_{33} \\
2 \varepsilon_{23} \\
2 \varepsilon_{13} \\
2 \varepsilon_{12}
\end{array}\right]\right)_{k}
$$

where the $c_{i j}$ are the transformed elastic coefficients referred to the $\left(x_{1}, x_{2}, x_{3}\right)$ coordinate system, which are related to the elastic coefficients in the material coordinates $\bar{c}_{i j}$, by

$$
\mathbf{C}_{k}=\left(\mathbf{T} \overline{\mathbf{C}} \mathbf{T}^{\mathrm{T}}\right)_{k}
$$

The transformation matrix $\mathbf{T}$ represents the transformation between the particular coordinate systems. This matrix depends directly on the layer orientation, $\theta_{k}$, where the index $k$ is referred to the $k$ th layer.

\subsection{PLATE/SHELL ELEMENT}

The shell element developed is based on the so-called Mindlin-Reissner plate theory where only $C^{0}$ continuity is required for the approximation of the kinematic variables. The element, called discrete shear triangle (DST) degenerates automatically [14] to the discrete kirchhoff triangle element (DKT) if the transverse shear effects are neglectable. The DST element is extended to the treatment of plate and shell structures with the incorporation of membrane stiffness [15]. The first-order theory leads to constant transverse shear stress, which violates equilibrium at the free surfaces of the plate and continuity requirements of the interlaminar shear stress. To account for the discrepancy between the constant state of shear strains in the first order theory and the quadratic or higher order distribution of shear strains in the elasticity, shear correction factors are introduced. These factors, calculated for laminated plates, are reported in different works that emphasize the use of exact or improved values [15]. The values collected in the literature are used in this work. In the Mindlin-Reissner, or first-order shear deformation laminated plate theory (FSDT), the assumption that the normal to the mid-surface of the undeformed plate remain straight, but not necessarily normal to the mid-surface of the deformed plate leads to the following definition of the displacement [16]

$$
\begin{aligned}
& u_{1}^{\prime \prime}\left(x_{m}^{\prime \prime}, t\right)=u_{1}^{\prime \prime 0}\left(x_{\alpha}^{\prime \prime}, t\right)+x_{3}^{\prime \prime} \phi_{1}^{\prime \prime}\left(x_{\alpha}^{\prime \prime}, t\right) \\
& u_{2}^{\prime \prime}\left(x_{m}^{\prime \prime}, t\right)=u_{2}^{\prime \prime 0}\left(x_{\alpha}^{\prime \prime}, t\right)+x_{3}^{\prime \prime} \phi_{2}^{\prime \prime}\left(x_{\alpha}^{\prime \prime}, t\right) \\
& u_{3}^{\prime \prime}\left(x_{m}^{\prime \prime}, t\right)=u_{3}^{\prime \prime 0}\left(x_{\alpha}^{\prime \prime}, t\right)
\end{aligned}
$$



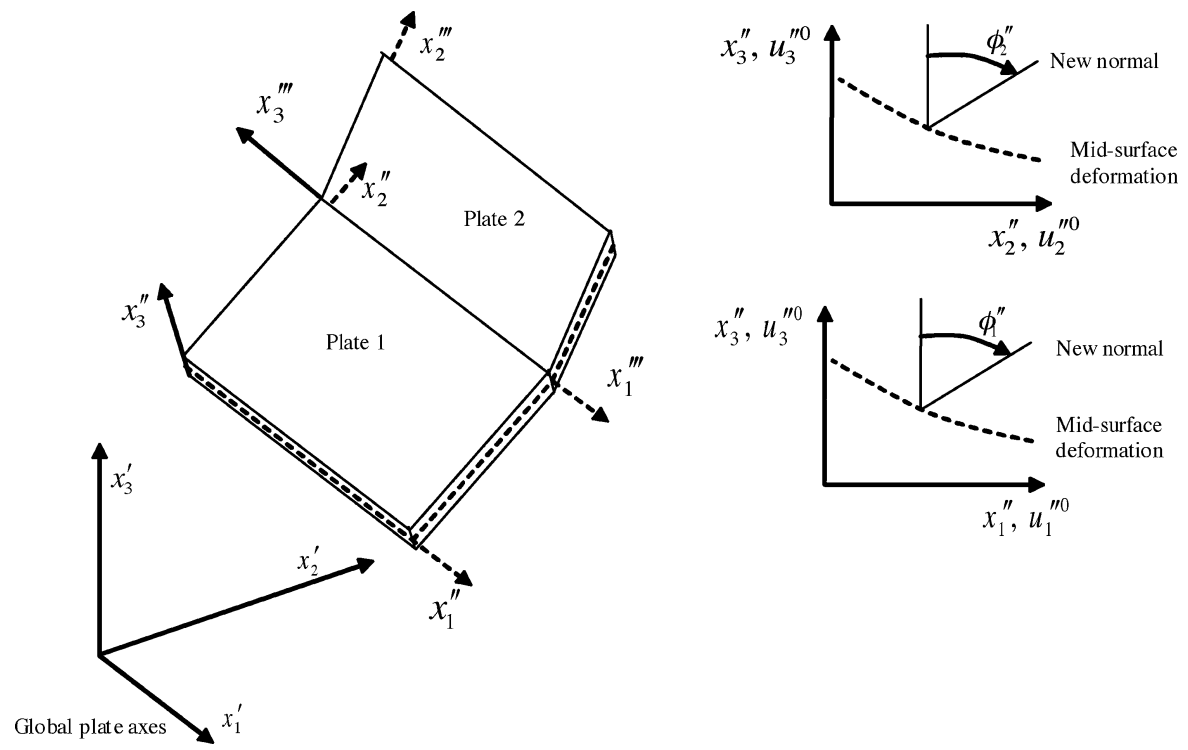

Figure 3. Global and local axes, local displacements for the Mindlin-Reissner theory.

where the Latin subscripts take the values 1,2 and 3 and the Greek subscripts take the values 1 and 2 . The quantities $u_{i}^{\prime \prime 0}$ represent the displacement component in the $x_{i}^{\prime \prime}$ direction for one point in the mid-surface and $\phi_{1}^{\prime \prime}$ and $-\phi_{2}^{\prime \prime}$ represent the rotations of the normal about the $x_{1}^{\prime \prime}$ and $x_{2}^{\prime \prime}$ axes, respectively, as seen in Figure 3.

The finite element developed is a triangular element with five degrees of freedom in each node, i.e., the nodal displacements $u_{1}^{\prime \prime 0}, u_{2}^{\prime \prime 0}$ and $u_{3}^{\prime \prime 0}$ and rotations $\phi_{1}^{\prime \prime}$ and $\phi_{2}^{\prime \prime}$, all expressed the element coordinate system. However, when one node is shared by elements with different orientations, the transformation of the displacements to a global system, with axes $x_{i}^{\prime}$, must be preformed before the element assemblage. Therefore, it is necessary to introduce a third rotation, $\phi_{3}^{\prime}$, in the global system, to account for the possible components projection of the rotations $\phi_{1}^{\prime \prime}, \phi_{2}^{\prime \prime}$ to the global system. In this context the relations between local and global components, at element level, take the form

$$
\mathbf{u}^{\prime \prime(e)}=\mathbf{R}^{(e)} \mathbf{u}^{(e)}
$$

with

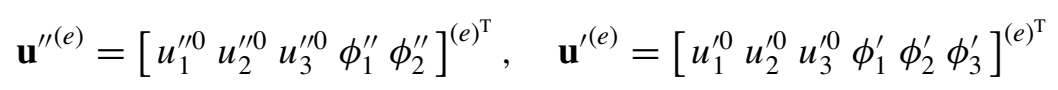

where $\mathbf{R}^{(e)}$ is the transformation matrix between the two systems. This relation is used only for non-coplanar nodes. For instance, if the local and global systems are 
paralel, the projection of the local rotations $\phi_{1}^{\prime \prime}, \phi_{2}^{\prime \prime}$ over the global axis $x_{3}^{\prime}$ is null. Therefore, for the nodes that are part of different elements, but are coplanar it is important to introduce a fictitious rotation at the local level to avoid a singularity in the stiffness matrix [17].

Using the Hamilton's Principle with appropriate interpolation functions [13] and following the standard displacement finite procedures, it is possible to obtain the global equilibrium system of linear equations in the form,

$$
\mathbf{M}_{f f} \ddot{\mathbf{q}}+\mathbf{K}_{f f} \mathbf{q}=\mathbf{F}
$$

At the element level $(e)$ the stiffness matrix expressed in the local coordinates $(\xi, \eta)$ is

$$
\mathbf{K}_{f f}^{(e)}=\int_{0}^{1} \int_{0}^{1-\eta}\left[\begin{array}{ccc}
\mathbf{B}_{m}^{\mathrm{T}} \mathbf{D}_{m} \mathbf{B}_{m} & \mathbf{B}_{m}^{\mathrm{T}} \mathbf{D}_{m b} \mathbf{B}_{b} & 0 \\
\mathbf{B}_{b}^{\mathrm{T}} \mathbf{D}_{b m} \mathbf{B}_{m} & \mathbf{B}_{b}^{\mathrm{T}} \mathbf{D}_{b} \mathbf{B}_{b} & 0 \\
0 & 0 & \mathbf{B}_{s}^{\mathrm{T}} \mathbf{D}_{s} \mathbf{B}_{s}
\end{array}\right]^{(e)}|\boldsymbol{J}| \mathrm{d} \xi \mathrm{d} \eta
$$

which is written in a compact form as

$$
\mathbf{K}_{f f}^{(\mathrm{e})}=\int_{0}^{1} \int_{0}^{1-\eta}\left(\mathbf{B}^{\mathrm{T}} \mathbf{D} \mathbf{B}\right)^{(\mathrm{e})}|\boldsymbol{J}| \mathrm{d} \xi \mathrm{d} \eta
$$

Matrix $\mathbf{B}$ represents the strain-displacement matrix, matrix $\mathbf{D}$ is the elasticity matrix and $|\mathbf{J}|$ is the determinant of the Jacobian matrix; which in triangular elements is the double of the element area. Subscripts $m, b$ and $s$ stand for membrane, bending and shear respectively, and

$$
\mathbf{B}^{(e)}=\mathbf{L} \mathbf{S}^{(e)}
$$

where $\mathbf{S}^{(e)}$ is the shape function matrix, which depends on the choice of finite element and on the laminate theory, and $\mathbf{L}$ is a matrix of differential operators. The constitutive equation for the laminate at element level is given by [14]

$$
\left[\begin{array}{l}
\mathbf{N} \\
\mathbf{M} \\
\mathbf{Q}
\end{array}\right]^{(\mathrm{e})}=\left[\begin{array}{ccc}
\mathbf{D}_{m} & \mathbf{D}_{m b} & 0 \\
\mathbf{D}_{b m} & \mathbf{D}_{b} & 0 \\
0 & 0 & \mathbf{D}_{s}
\end{array}\right]^{(\mathrm{e})}\left[\begin{array}{c}
\varepsilon^{0} \\
\varepsilon^{i} \\
\gamma^{s}
\end{array}\right]^{(\mathrm{e})}
$$

Sub-matrix $\mathbf{D}_{m}$ relates resultant membrane forces $\mathbf{N}$ to membrane strain $\varepsilon^{0}$, sub-matrix $\mathbf{D}_{b}$ relates generalized moments $\mathbf{M}$ to generalized curvature $\varepsilon^{i}$, submatrix $\mathbf{D}_{m b}$ relates resultant membrane forces to generalized curvature, and the sub-matrix $\mathbf{D}_{s}$ relates resultant transverse shear $\mathbf{Q}$ to the shear strains $\gamma^{s}$. As each layer may have different properties, the elasticity matrix $\mathbf{D}$ must be evaluated by a 


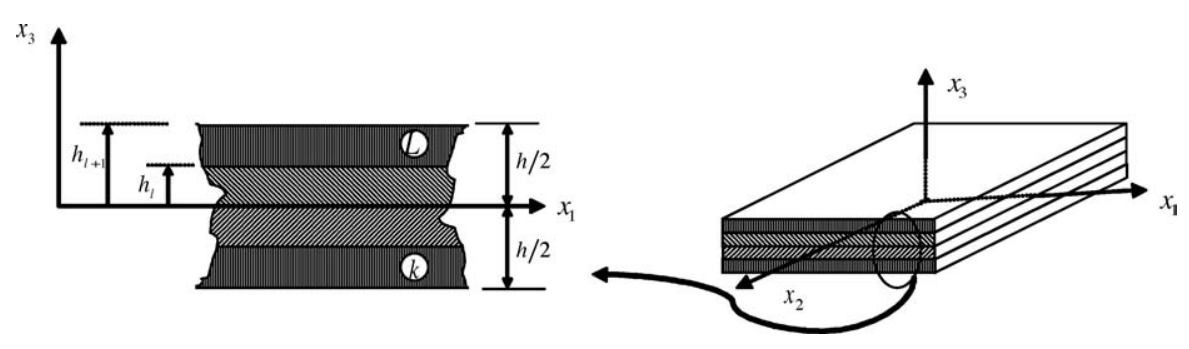

Figure 4. Coordinate system and layer numbering used for a typical laminated plate.

summation carried out over all the element thickness. Therefore, equivalent single layer theories produce the equivalent stiffness matrix as a weighted average of the individual layer stiffness through the thickness. These matrices are dependent on each layer orientation and are given as

$$
\begin{aligned}
& \left(\mathbf{D}_{m}, \mathbf{D}_{b}, \mathbf{D}_{m b}, \mathbf{D}_{s}\right)=\sum_{k=1}^{n}\left(\mathbf{D}_{m}, \mathbf{D}_{b}, \mathbf{D}_{m b}, \mathbf{D}_{s}\right)_{k} \\
& =\sum_{k=1}^{n}\left(\left[\begin{array}{lll}
c_{11} & c_{12} & c_{16} \\
c_{21} & c_{22} & c_{26} \\
c_{61} & c_{62} & c_{66}
\end{array}\right] H_{1},\left[\begin{array}{lll}
c_{11} & c_{12} & c_{16} \\
c_{21} & c_{22} & c_{26} \\
c_{61} & c_{62} & c_{66}
\end{array}\right] H_{2},\left[\begin{array}{lll}
c_{11} & c_{12} & c_{16} \\
c_{21} & c_{22} & c_{26} \\
c_{61} & c_{62} & c_{66}
\end{array}\right] H_{3},\right. \\
& \left.\left[\begin{array}{ll}
c_{44} & c_{45} \\
c_{54} & c_{55}
\end{array}\right] H_{1}\right)_{k}
\end{aligned}
$$

with,

$$
H_{n}^{=} \int_{h_{l-1}}^{h_{l}}\left(x_{3}^{n-1}\right) \mathrm{d} z=\frac{1}{n}\left(h_{l+1}^{n}-h_{l}^{n}\right)
$$

where $h_{i}$ is defined in Figure 4. The axis $x_{3}$ is taken positive upward from the mid-plane. The $L$ th layer is located between the points $x_{3}=h_{l}$ and $x_{3}=h_{l+1}$ in the direction of the thickness.

At the $(e)$ element level the consistent mass matrix is given in local coordinates $(\xi, \eta)$ by

$$
\mathbf{M}_{f f}^{(\mathrm{e})}=\int_{0}^{1} \int_{0}^{1-\eta} \rho^{(\mathrm{e})}\left(\mathbf{S}^{\mathrm{T}} \mathbf{m} \mathbf{S}\right)^{(\mathrm{e})}|\boldsymbol{J}| \mathrm{d} \xi \mathrm{d} \eta
$$

where $\mathbf{m}$ is the matrix that has the inertial terms and $\rho$ is the specific mass of the element. A diagonalization procedure is used to obtain the lumped mass matrix [18]. 


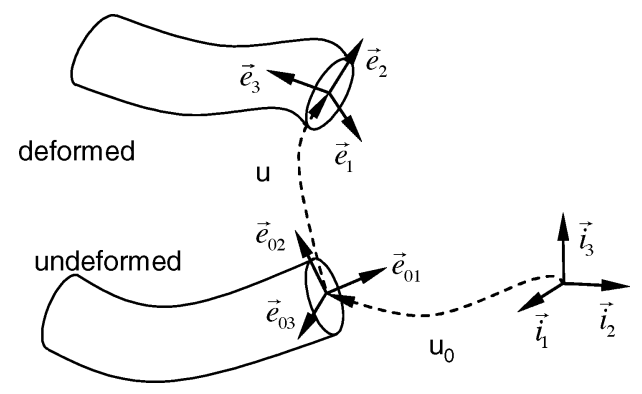

Figure 5. Beam local coordinate frames and point displacements.

\subsection{DISPLACEMENT AND STRAIN FIELDS OF THE BEAM}

In the finite element analysis of the 1-D beam problem the Timoshenko first-order shear deformation theory (FSDT) is used. The Euler-Bernoulli theory ignores transverse shear deformation, which has a significant effect on the behavior of the fiber reinforced laminated structures due to the large difference in the elastic properties between fiber and matrix materials. This leads to high ratios of in-plane Young's modulus to transverse modulus for most applications. When these high ratios are coupled with depth effects, this theory is inadequate for the analysis of highly anisotropic beams. To improve the situation, the Timoshenko FSDT is applied to multilaminated anisotropic beams. In this theory, the transverse strains are constants throughout the depth of the beam, hence, the transverse shears are also constant. This discrepancy is overcome by introducing shear correction factors.

Consider a set of unit vectors $\mathbf{e}_{1}, \mathbf{e}_{2}$, and $\mathbf{e}_{3}$ attached to the centroid of the beam cross-section, being $\mathbf{e}_{1}$ aligned with the beam axis while $\mathbf{e}_{2}$ and $\mathbf{e}_{3}$ define the plane of the cross-section, as depicted by Figure 5. Let $u_{1}\left(x_{1}, x_{2}, x_{3}, t\right), u_{2}\left(x_{1}, x_{2}, x_{3}, t\right)$ and $u_{3}\left(x_{1}, x_{2}, x_{3}, t\right)$ be components of the displacement of an arbitrary point of the beam in the $\mathbf{e}_{1}, \mathbf{e}_{2}$, and $\mathbf{e}_{3}$ directions respectively. The displacement field in the plane of the cross-section is described by,

$$
\begin{aligned}
& u_{1}\left(x_{m}, t\right)=u_{1}^{0}\left(x_{1}, t\right)+x_{3} \phi_{2}\left(x_{1}, t\right)-x_{2} \phi_{3}\left(x_{1}, t\right) \\
& u_{2}\left(x_{m}, t\right)=u_{2}^{0}\left(x_{1}, t\right)-x_{3} \phi_{1}\left(x_{1}, t\right) \\
& u_{3}\left(x_{m}, t\right)=u_{3}^{0}\left(x_{1}, t\right)+x_{2} \phi_{1}\left(x_{1}, t\right)
\end{aligned}
$$

where $u_{i}^{0}$ is the displacement of the centroid in the direction $x_{i}$ and $\phi_{1}$ is the angle of twist of the cross-section about $x_{1}$. The rotations of the cross-sections $\phi_{2}\left(x_{1}, \mathrm{t}\right)$ and $\phi_{3}\left(x_{1}, \mathrm{t}\right)$ are positive about axes $\mathbf{e}_{2}$ and $\mathbf{e}_{3}$, respectively.

Using the Hamilton's Principle with the appropriate interpolation functions [14] and following the standard displacement finite procedures, it is possible to obtain the equilibrium system of linear equations in the form of Equation (24). At the (e) 
element level the consistent mass matrix is given in the local coordinate $(\xi)$ by

$$
\mathbf{K}_{f f}^{(e)}=\int_{-1}^{1}\left(\mathbf{B}^{\mathrm{T}} \mathbf{D} \mathbf{B}\right)^{(e)}|\boldsymbol{J}| \mathrm{d} \xi
$$

where all the quantities involved have been defined in Section (3.2). The matrix D is obtained by using the code VABS that is a standard 2-D Finite Element code that can compute the stiffness tensor $\mathbf{D}$ for arbitrary materials and geometries [19]. According to $\mathbf{Y u}$ and Hodges[19] for the Timoshenko theory the matrix $\mathbf{D}$ is express as

$$
\mathbf{D}=\left[\begin{array}{cc}
\mathbf{X} & \mathbf{F} \\
\mathbf{F}^{\mathrm{T}} & \mathbf{G}
\end{array}\right]
$$

where the sub-matrix $\mathbf{X}$ relates the resultant axial force $\mathbf{N}_{x x}$ with the axial strain and the generalized curvatures, and the generalized moments $\mathbf{M}=\left[\begin{array}{ll}M_{x x} & M_{y y} M_{z z}\end{array}\right]^{\mathrm{T}}$ with the axial strain and the generalized curvatures. The sub-matrix $\mathbf{F}$ relates the resultant axial force and the generalized moments with the transverse shear, while the sub-matrix $\mathbf{G}$ relates the resultant transverse shear force with the shear strains $\boldsymbol{\gamma}=\left[2 \varepsilon_{12} 2 \varepsilon_{13}\right]^{\mathrm{T}}$. These matrices are computed using a standard 2-D Finite Element code over the section of the beam. In the analysis of the beam cross-section, the classical $w_{0}$ and the first-order warping $w_{1}$ fields are discretized as

$$
\begin{aligned}
& w_{0}\left(x_{1}, x_{2}, x_{3}\right)=\mathbf{N}\left(x_{2}, x_{3}\right) \mathbf{V}_{0}\left(x_{1}\right) \\
& w_{1}\left(x_{1}, x_{2}, x_{3}\right)=\mathbf{N}\left(x_{2}, x_{3}\right) \mathbf{V}_{1}\left(x_{1}\right)
\end{aligned}
$$

where $\mathbf{N}$ represents the element shape functions and $\mathbf{V}_{0}$ and $\mathbf{V}_{1}$ represents the column matrices of the nodal values of the warping displacements over the crosssection. First the expressions (37) and (38) are substituted in the expression for the strain energy of the cross-section the warping field. Then the minimization of this energy is done with respect to the warping field. The result of using the variational-asymptotic method of Berdichevsky is that the warping functions can be determined asymptotically in terms of 1-D strain measures as

$$
\begin{aligned}
& \mathbf{V}_{0}=\hat{\mathbf{V}}_{0} \varepsilon \\
& \mathbf{E} \hat{\mathbf{V}}_{0}=\left(\mathbf{H} \boldsymbol{\psi}_{c l} \boldsymbol{\psi}_{c l}^{\mathrm{T}}-\mathbf{I}\right) \mathbf{C}_{h \varepsilon}
\end{aligned}
$$

After the classical warping calculation the first order warping functions can be computed using an identical procedure, as

$$
\begin{aligned}
& \mathbf{V}_{1}=\hat{\mathbf{V}}_{1} \varepsilon^{\prime} \\
& \mathbf{E} \hat{\mathbf{V}}_{1}=\left(\mathbf{I}-\mathbf{H} \Psi_{c l} \mathbf{\Psi}_{c l}^{\mathrm{T}}\right)\left[\mathbf{C}_{\varepsilon l}^{\mathrm{T}}+\left(\mathbf{C}_{h l}^{\mathrm{T}}-\mathbf{C}_{h l}\right) \hat{\mathbf{V}}_{0}\right]
\end{aligned}
$$


where matrices $\mathbf{E}, \mathbf{C}_{h \varepsilon}, \mathbf{C}_{h l}, \mathbf{C}_{l l}$, and $\mathbf{C}_{\varepsilon l}$ contains information on the material properties on the geometry of a given cross-section. After the computation of the warping functions and their substitution in the strain energy matrices $\mathbf{G}, \mathbf{F}$ and $\mathbf{X}$ are computed as

$$
\begin{aligned}
& \mathbf{G}=\mathbf{A}^{*} \mathbf{R}^{-1} \mathbf{A}^{* \mathrm{~T}} \\
& \mathbf{F}=-\mathbf{B}^{\mathrm{T}} \mathbf{A}^{*-1} \mathbf{G} \quad ; \quad \mathbf{B}=\left(\hat{\mathbf{V}}_{0}^{\mathrm{T}} \mathbf{C}_{h l}+\mathbf{C}_{\varepsilon l}\right) \hat{\mathbf{V}}_{0} \\
& \mathbf{X}=\mathbf{A}+\mathbf{F G}^{-1} \mathbf{F}^{\mathrm{T}}
\end{aligned}
$$

where the matrices $\mathbf{A}$ and $\mathbf{R}$ are given by

$$
\begin{aligned}
& \mathbf{A}=\mathbf{C}_{\varepsilon \varepsilon}+2 \hat{\mathbf{V}}_{0}^{\mathrm{T}} \mathbf{C}_{h \varepsilon} ; \quad \mathbf{A}^{*}=-\left[\begin{array}{cc}
0 & 0 \\
0 & 0 \\
0 & -1 \\
1 & 0
\end{array}\right] \mathbf{A} \\
& \mathbf{R}=\hat{\mathbf{V}}_{0}^{\mathrm{T}} \mathbf{C}_{l l} \hat{\mathbf{V}}_{0}+2 \hat{\mathbf{V}}_{1}^{\mathrm{T}} \mathbf{C}_{h l} \hat{\mathbf{V}}_{0}
\end{aligned}
$$

For more details on the calculation of these matrices the reader is referred to reference [20].

\section{Application Cases}

\subsection{THE ACTUATED BEAM PROBLEM}

This example consists in a cantilevered beam that is actuated at its mid-point by the crank link mechanism represented in Figure 6, which is based on the demonstrative

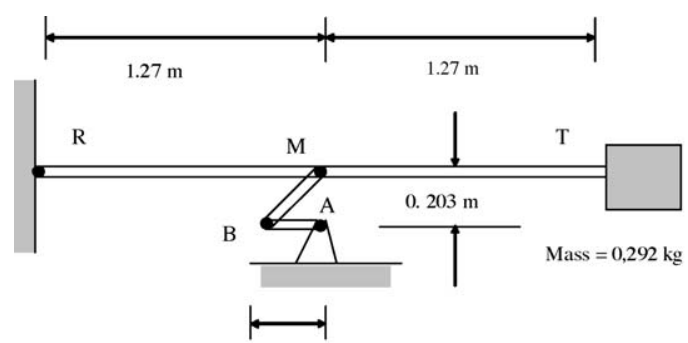

0. $203 \mathrm{~m}$

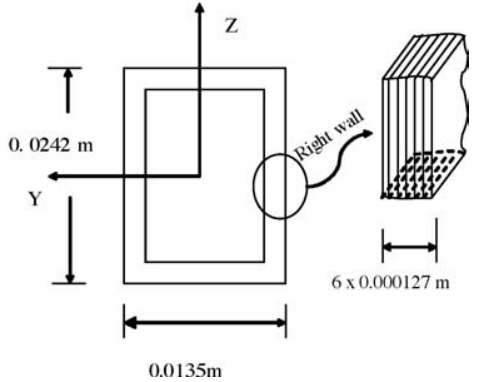

$0.0135 \mathrm{~m}$

Figure 6. The actuated beam problem. 
Table I. Characteristics of the three lay-ups considered in the applications of the actuated beam.

\begin{tabular}{lllll}
\hline Lay-up type & Upper wall & Lower wall & Left wall & Right wall \\
\hline Lay-up 1 & $\left(0^{0}\right)_{6}$ & $\left(0^{0}\right)_{6}$ & $\left(0^{0}\right)_{6}$ & $\left(0^{0}\right)_{6}$ \\
Lay-up 2 & $\left(30^{0} / 0^{0}\right)_{3}$ & $\left(30^{0} / 0^{0}\right)_{3}$ & $\left(30^{0} / 0^{0}\right)_{3}$ & $\left(30^{0} / 0^{0}\right)_{3}$ \\
Lay-up 3 & $\left(15^{0}\right)_{6}$ & $\left(-15^{0}\right)_{6}$ & $\left(-15^{0} / 15^{0}\right)_{3}$ & $\left(15^{0} / 15^{0}\right)_{3}$ \\
\hline
\end{tabular}

example provided by Bauchau and Hodges [7]. The simulation model topology uses revolute joints in points $\mathrm{A}, \mathrm{B}$ and $\mathrm{R}$, and a spherical joint in point $\mathrm{M}$. A tip mass is located at the end of the beam. The crank is a rigid body with a constant angular velocity of $3.14 \mathrm{rad} / \mathrm{sec}$. The beam and the link are modeled using the finite element method. The cross-sections of the beam and the link consist in one thin-walled, rectangular section depicted in Figure 6, with the dimensions of $0.0242 \times 0.00136$ $\mathrm{m}$. The cross-section walls consist in six layers of graphite/epoxy material with the material properties $E_{l}=142 \mathrm{GPa}$, a transverse modulus of $E_{t}=9.790 \mathrm{GPa}$, a shearing modulus of $G_{l T}=5.998 \mathrm{GPa}$, and a Poisson's ratio of 0.42 . In this application, it is considered that a lay-up of zero degrees, i.e., the fibers are aligned with the axis of the beam.

The beam cross-section is modeled using the finite element procedure VABS. The sectional properties are computed for several fiber orientations with respect to the axis of the beam, as shown in Table I.

The modal superposition technique, described in this work, using four modes of vibration, is used to reduce the number of generalized flexible coordinates. Two multibody models of the system are simulated. In the first model, the beam and the link were modeled with eight and two Timoshenko beams elements respectively. In the second model, the beam is modeled with 320 shell elements and the link with two beam elements. For both models the theory used is the Timoshenko beam/plate theory.

The response of the system is simulated for a time period of $6 \mathrm{sec}$. The tip transverse deflection of the beam obtained with beam model is represented in Figure 7. The difference between the solutions obtained with the different lay-ups is noticeable. The difference is larger between lay-up one and the others two. This is justified by the fact that the sectional properties of the beam are stiffer in the case of lay-up one.

In the second model, the lay-up one and three are simulated with the shell elements. The solution obtained is represented in Figure 8. The simulations using shell elements do not show larger differences in the deformations for the actuated beams with lay-ups one and three. The instant where the maximum deformation is achieved it is very different in the various arrangements. Furthermore, it is observed that the qualitative response of the system is similar to that obtained with the beam elements but the amplitude of the vibration is smaller, suggesting a stiffer cantilever beam. 


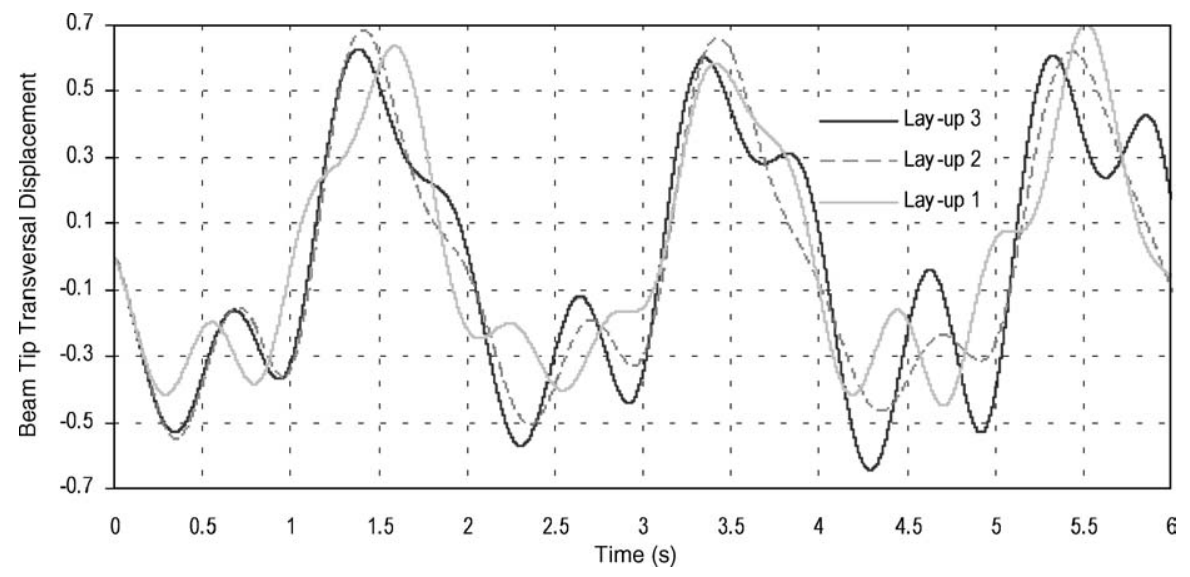

Figure 7. Tip transverse deflection of the beam considering beam elements.

\subsection{THE DOUBLE PENDULUM}

This example consists of a double pendulum made of two flexible beams that have tip point masses at their ends and that are connected by revolute joints. The initial positions of the system bodies are such that they are aligned in the horizontal position, as shown in Figure 9. Table II presents the geometrical characteristics of the model and also the material properties used. In the case simulated the arm beam rotates with a constant angular velocity of $20 \mathrm{rad} / \mathrm{sec}$.

The dimensionless deflection of the mid-node of the arm and hand obtained in the simulation of the double pendulum model where the beams are made of

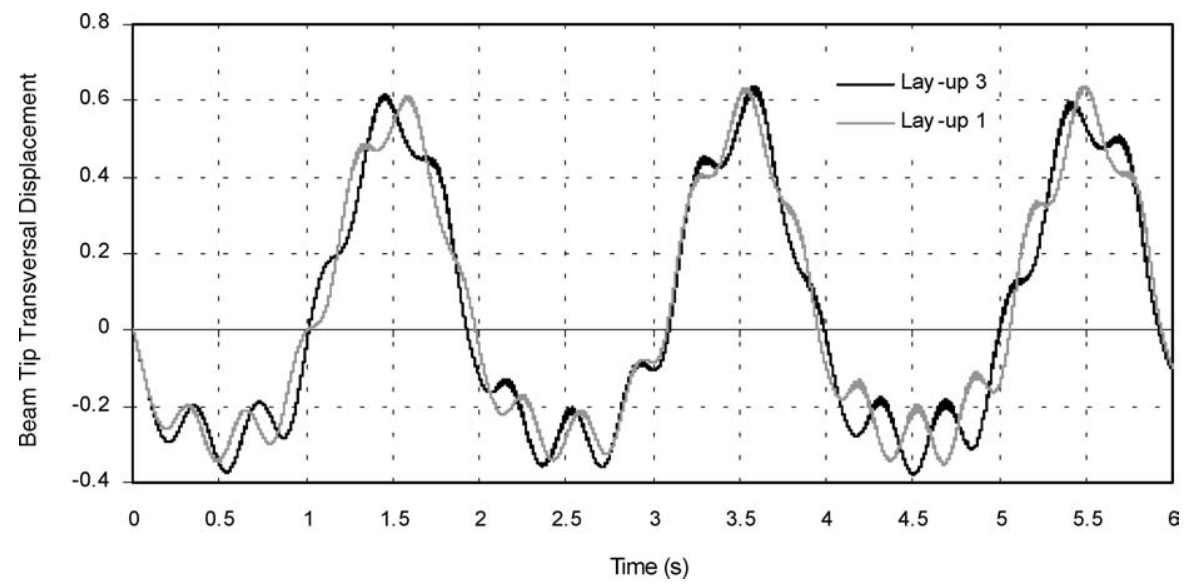

Figure 8. Tip transverse deflection of the beam considering shell elements. 


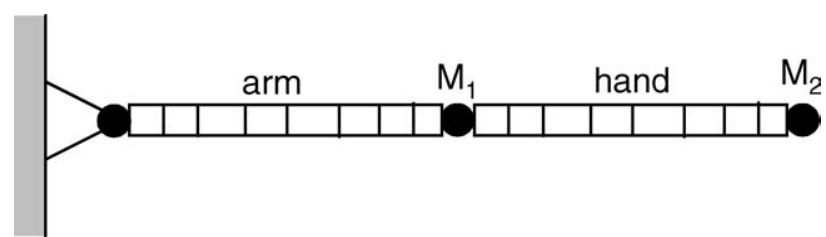

Figure 9. Initial position of the double pendulum.

Table II. Characteristics of the double pendulum.

\begin{tabular}{|c|c|c|c|c|}
\hline & Arm & Hand & Isotropic material & Composite material \\
\hline & & & & $\mathrm{E}_{2}=\mathrm{E}_{3}=\mathrm{E}_{1}=129,207 \mathrm{GPa}$ \\
\hline Length $(L)$ & $0.545 \mathrm{~m}$ & $0.675 \mathrm{~m}$ & $E=73 \mathrm{GPa}$ & $\mathrm{G}_{12}=5.15658 \mathrm{GPa} \mathrm{G}_{23}=2.5414 \mathrm{GPa}$ \\
\hline $\mathrm{b}$ & $0.06 \mathrm{~m}$ & $0.04 \mathrm{~m}$ & $\rho=2700 \mathrm{~kg} / \mathrm{m}^{3}$ & $\mathrm{G}_{13}=4.3053 \mathrm{GPa} v_{12}=v_{13}=0.3$ \\
\hline $\mathrm{h}$ & $0.015 \mathrm{~m}$ & $0.01 \mathrm{~m}$ & & $\nu_{23}=0.218837 \rho=1550.066 \mathrm{~kg} / \mathrm{m}^{3}$ \\
\hline Total mass & & & & \\
\hline arm & & & $1.324 \mathrm{~kg}$ & $0.729 \mathrm{~kg}$ \\
\hline hand & & & $0.760 \mathrm{~kg}$ & $0.418 \mathrm{~kg}$ \\
\hline Point mass $\mathrm{M}_{1}$ & \multicolumn{2}{|c|}{$1 \mathrm{~kg}$} & & \\
\hline Point mass $\mathrm{M}_{2}$ & \multicolumn{2}{|c|}{$3 \mathrm{~kg}$} & & \\
\hline
\end{tabular}

isotropic material are displayed in Figures 10 and 11. The output parameter is a dimensionless value obtained as the ratio of the beam mid-node deflection with respect to the body fixed coordinate frame by the half beam length. The multibody model of the double pendulum uses four vibration modes in order to reduce the number of generalized elastic coordinates, using the mode component synthesis.

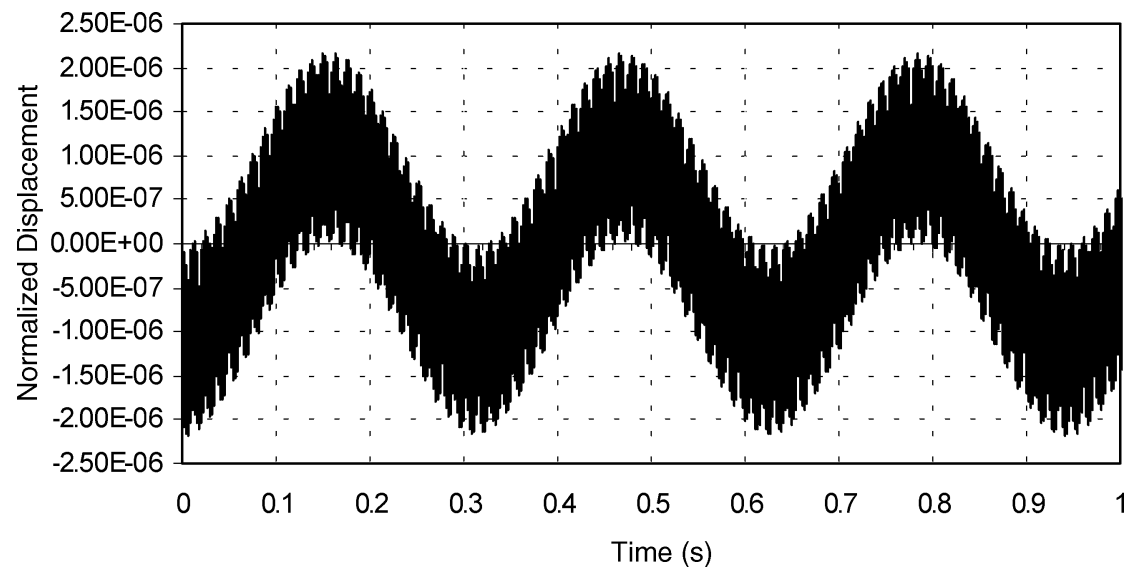

Figure 10. Double pendulum arm mid-node dimensionless deflection with isotropic material. 


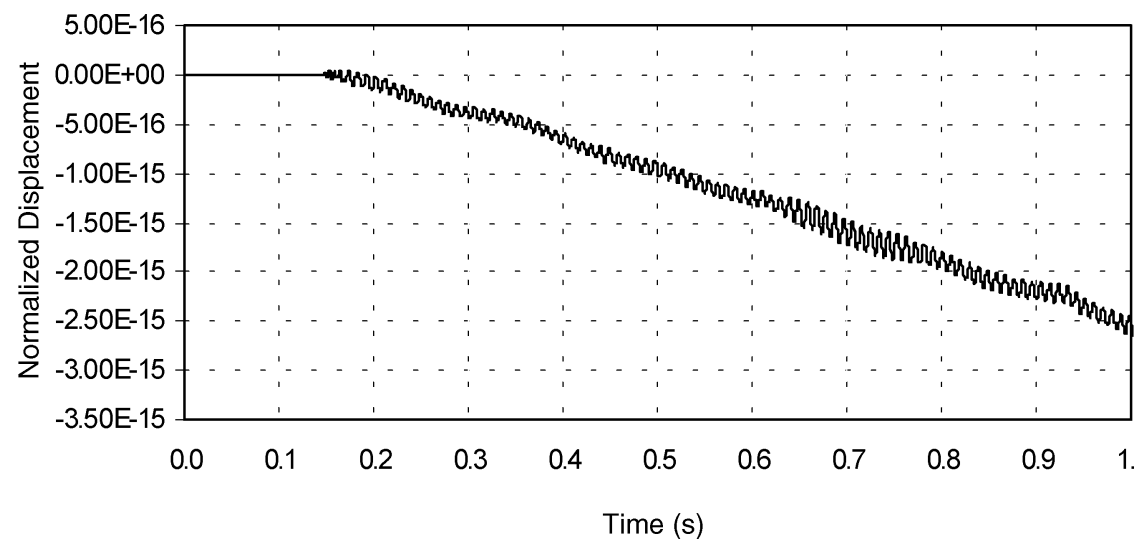

Figure 11. Double pendulum hand mid-node dimensionless deflection with isotropic material.

The deformation of the arm is several orders of magnitudes higher than the deformations of the hand. The hand can be represented by a rigid body model when the isotropic material is used because the hand displacement field is almost null.

The same multibody model is used to simulate a double pendulum where the beams are made of a composite material, with the characteristics shown in Table II. The first simulation with composite material is equivalent to the lay-up one because the fibers are aligned with the axis of the beam, $0^{\circ}$. The response of the mid-point of the arm and hand are represented in Figures 12 and 13, respectively.

With the composite materials the weight of the arm and hand is lower than for the model that uses the isotropic material. In this case, the arm has a lower deformation than the deformation observed for the arm made of isotropic material. However, the deformation of the hand for the case of the double pendulum made of composite

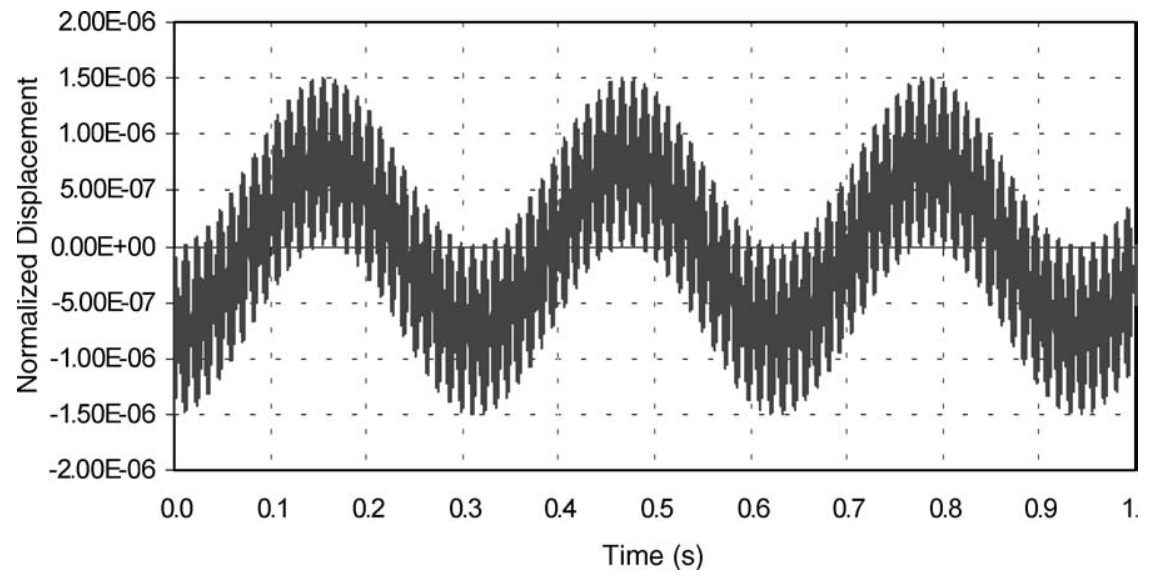

Figure 12. Double pendulum arm mid-node dimensionless deflection with composite material lay-up 1. 


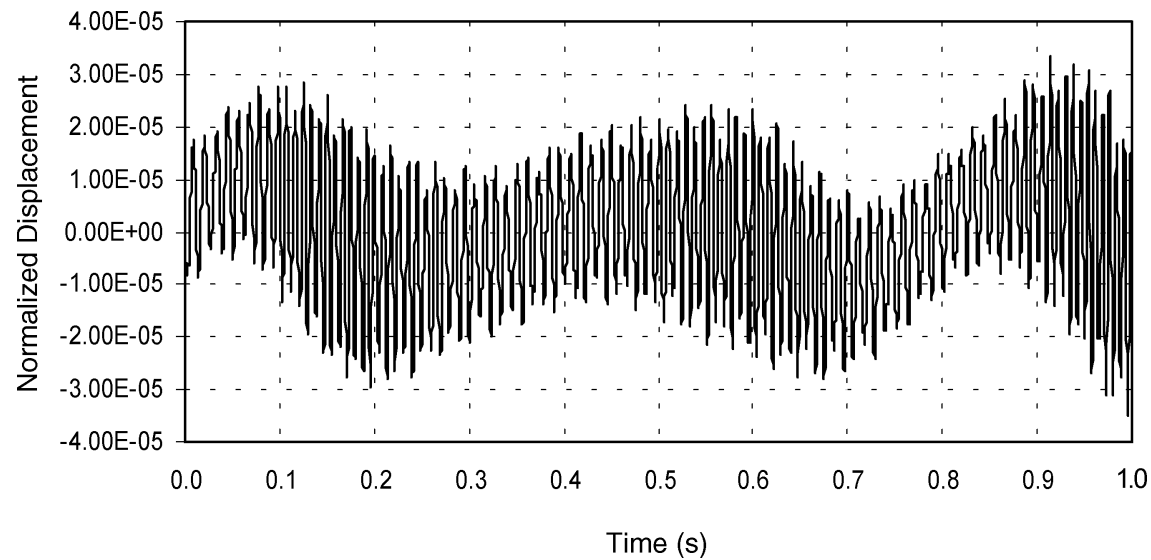

Figure 13. Mid-node dimensionless deflection of the double pendulum Hand with composite material lay-up 1.

material has a magnitude similar to that observed for the arm. When a composite material is used for the double pendulum, it is not possible to represent the hand by a rigid body as it is suggested for the isotropic material.

The second simulation with composite material corresponds to lay-up two. In this case, the fibers are not aligned with the axis of the beam but they form an angle of $30^{\circ}$ with the axis of the beam. The response of the mid-point of the arm and hand are represented in Figures 14 and 15 respectively.

When the fiber orientations change the response of the simulation also change, as expected. In the arm, the change of lay-up does not have a large influence in the magnitude of the deformation as seen in Figures 12 and 14. The deformation value for lay-up 2 is only two times larger than for lay-up 1. According to Figures 13 and 15, when the lay-up 2 is used for the hand the deformation is almost ten

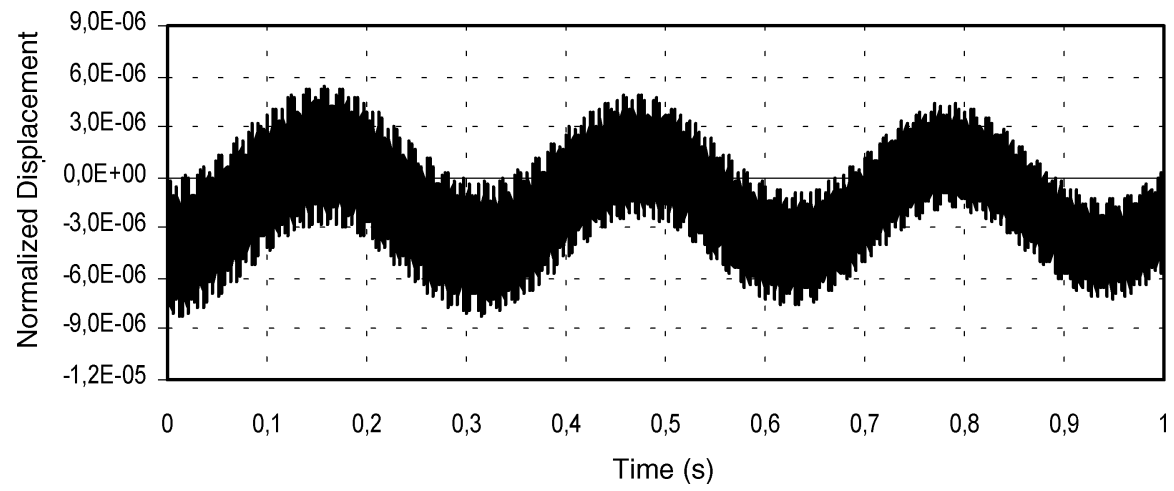

Figure 14. Mid-node dimensionless deflection of the double pendulum arm with composite material lay-up 2. 


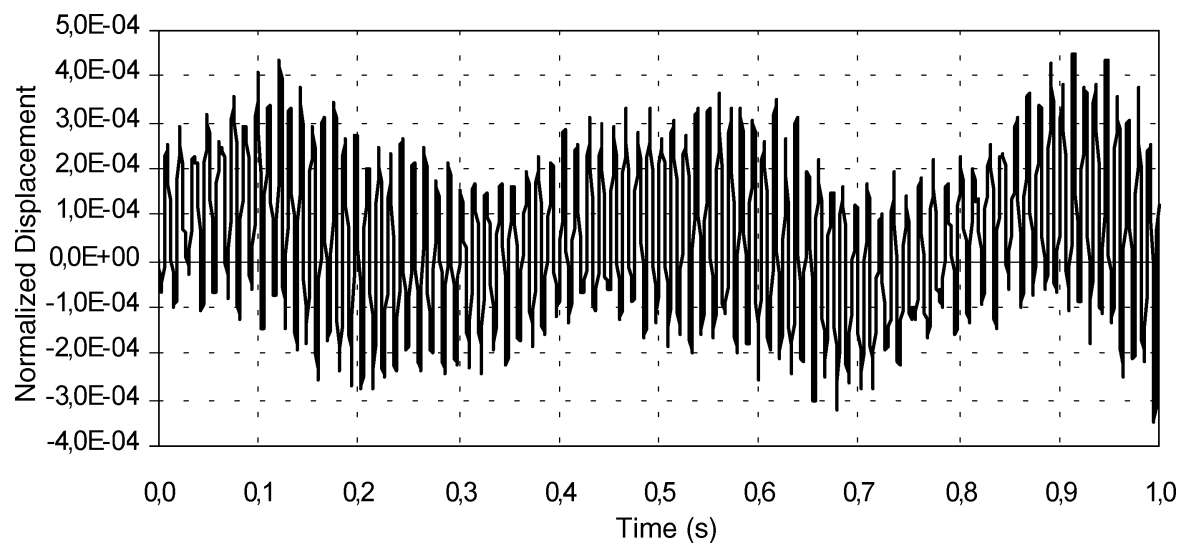

Figure 15. Mid-node dimensionless deflection of the double pendulum Hand with composite material lay-up 2.

times larger than the deformation associated with the lay-up 1. Thus, it is possible to observe in this example the behavior associated with composite materials, i.e. for the same material properties of individual lamina, the lamination scheme can be changed in order to provide the stiffness and strength of the laminate that satisfy the structural requirements. The behavior of one lamination scheme is very dependent on the solicitation it is subjected to.

\section{Conclusions}

A multibody-based methodology for the analysis of flexible multibody systems made of composite materials was described in this work. The formulation used a finite element based description of the flexibility of each body referred to a body fixed coordinate system. Special emphasis was put in the representation of beam elements made of composite materials where a three step procedure, proposed by Bauchau and Hodges [7], was used. This procedure consists in calculating the cross-sectional properties of the beam based on a planar finite element analysis of the cross-section, followed by the simulation of the flexible multibody system and finalized with the recovery of the three-dimensional beam stresses, if required. Within the local body fixed coordinate system the deformations of the bodies are considered to be small. Furthermore, the material models used in the foreseen applications of the methodology are limited to be linear elastic. With these assumptions the mode component synthesis can be used in the flexible multibody formulation in order to reduce the number of equilibrium equations used to solve the problem.

The application of the methodology was demonstrated through two numerical examples consisting in an actuated cantilever beam and a double pendulum. The results were analyzed with reference to the different multibody models that can be used, this is, the complete model and the reduced model that uses the mode 
component synthesis and also with reference to the use of isotropic and composite materials. These preliminary results show that the formulation proposed is effective offering a good potential to be applied to more complex cases of structures made of composite materials and experiencing large rigid body rotations.

\section{References}

1. Shabana, A., 'Dynamic analysis of large-scale inertia variant flexible systems', Ph.D. Thesis, University of Iowa, Iowa City, Iowa, 1982.

2. Ambrósio, J. and Gonçalves, J. 'Complex flexible multibody systems with application to vehicle dynamics', Multibody System Dynamics 6(2), 2001, 163-182.

3. Nikravesh, P., Computer-Aided Analysis of Mechanical Systems, Prentice Hall, EnglewoodCliffs, New Jersey, 1988.

4. Haug, E., Computer Aided Kinematics and Dynamics of Mechanical Systems, Allyn and Bacon, Boston, Massachussetts, 1989.

5. de Jalón, J. and Bayo, E. Kinematic and Dynamic Simulation of Multibody Systems : The RealTime Challenge, Springer-Verlag, New York, 1994.

6. Gonçalves, J. and Ambrósio, J., 'Advanced modeling of flexible multibody dynamics using virtual bodies', Computer Assisted Mechanics and Engineering Sciences 9(3), 2002, 373-390.

7. Bauchau, O. and Hodges, D. 'Analysis of nonlinear multibody systems with elastic couplings', Multibody System Dynamics 3, 1999, 163-188.

8. Cesnik, C. and Hodges, D., 'VABS: A new concept for composite rotor blade cross-sectional modeling', Journal of the American Helicopter Society 42(1), 1997, 27-38.

9. Yoo, W.S. and Haug, E., 'Dynamics of flexible mechanical systems using vibration and static correction modes', Journal of Mechanisms, Transmissions and Automation in Design 108, 1986, 315-322.

10. Pereira, M. and Proença, P., 'Dynamic analysis of spatial flexible multibody systems using joint coordinates', International Journal for Numerical Methods in Engineering 32, 1991, 1799-1812.

11. Cavin, R. and Dusto, A., 'Hamilton's principle: Finite element method and flexible body dynamics', AIAA Journal 15(12), 1977, 1684-1690.

12. Hodges, D., 'A review of composite rotor blade modeling', AIAA Journal 28(3), 1990, 561-565.

13. Reddy, J., Mechanics of Laminated Composite Plates: Theory and Analysis, CRC Press, Boca Raton, 1997.

14. Batoz, J. and Lardeur, P., 'Adiscrete shear triangular nine d.o.f. element for the analysis of thick to very thin plates', International Journal for Numerical Methods in Engineering 28, 1989, 533-560.

15. Lardeur, P., Development e evaluation de Deux elements finits de plaque e coques composites avec influence du cisalhement transversal Ph.D. Thesis, Université de Technologie de Compiègne, France, 1990.

16. Ochoa, O. and Reddy, J., Finite Element Analysis of Composite Laminates, Kluwer AcademicPublishers, Dordrecht, The Netherlands, 1992.

17. Oñate, E., Cálculo de Estruturas por el Método de Elementos Finitos, Segunda Edicion, Centro International de Métodos Numéricos en Ingeniería, Barcelona, Spain, 1995.

18. Cook, R.., Concepts and Applications of Finite Element Analysis, 2nd edn. Wiley and Sons, New York, 1987.

19. Yu, W. and Hodges, D., 'On Timoshenko-like modeling of initially curved and twisted composite beams', International Journal of Solids and Structures 39, 2002, 5101-5121.

20. Popescu, B. and Hodges, D. 'On asymptotically correct Timoshenko-like anisotropic beam theory', International Journal of Solids and Structures 37, 2000, 535-558. 